\title{
High-order Harmonic Generation
}

\author{
Krzysztof Jakubczak \\ Institute of Physics, Academy of Sciences of the Czech Republic \\ Czech Republic
}

\section{Introduction}

X-rays were observed for the first time by Wilhelm Conrad Röntgen in 1895 (Röntgen, 1895). During the first century since that great event X-rays were benefiting mostly from their spatial resolution capability. However, recently it was possible to take advantage also from their temporal resolution due to novel sources providing ultrashort bursts of shortwavelength radiation (i.e. wavelength $\lambda<100 \mathrm{~nm}$ ) and to get an inside view of physical processes in molecules and atoms. One possibility of how to obtain ultrashort bursts of coherent extreme ultraviolet (abbreviated XUV or EUV; wavelength spectral range between $10-100 \mathrm{~nm})$, soft X-ray $(1-10 \mathrm{~nm})$ and/or X-ray radiation $(<1 \mathrm{~nm})$ is by high-order harmonic generation (HHG) process. It involves interaction of laser light at a given frequency during which it is being converted into integer multiples of the fundamental frequency through a highly nonlinear interaction with a conversion medium (typically a noble gas; Brabec \& Krausz, 2000). Laser-driven HHG uses acceleration of electrons on time-scales that are of the order of an optical cycle of the laser field. Currently this technique gives rise to the shortest flashes of light ever generated in a laboratory which are typically of the order of a few hundreds of attoseconds ( 1 as $=10^{-18}$ s; Paul et al., 2001; Kienberger et al., 2004; Schultze et al., 2007). When laser field of intensity of about $10^{14}-10^{15} \mathrm{~W} / \mathrm{cm}^{2}$ and time durations in

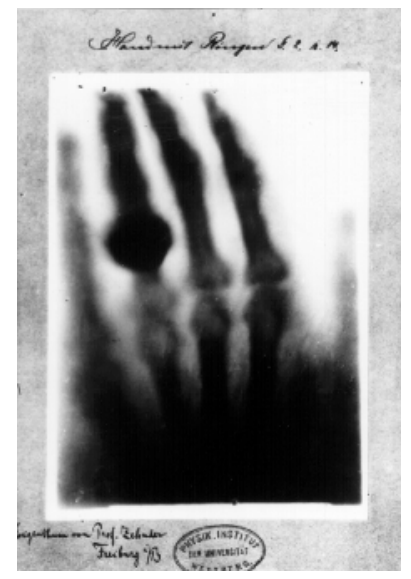

Fig. 1. Ms. Röntgen's hand. First medical imaging with X-rays (December 22, 1985; source: wikipedia.org). 
range of femtoseconds ( $1 \mathrm{fs}=10^{-15} \mathrm{~s}$ ) is applied to the gas, a plateau of equally intense harmonics of very high order can be observed. The atom is ionized when the absolute electric field of the laser is close to its crest during an optical cycle and is pulled away from the parent ion. Since the laser electric field changes its sign about a quarter of a period later, the electron will slow down, stop at a position far from the ion and start to accelerate back towards it (Corkum, 1993). When it returns to the ion, it can possess significant amount of kinetic energy, much larger than the photon energy but being its multiple. This energy plus the ionization potential is transferred into emitted photon energy as soon as the electron recombines with its parent ion, which gives rise to very high harmonic orders observed in the experiments (Macklin, 1993). Thus HHG represents a source of coherent X-rays bursts of ultrashort time duration. Additionally, the HHG source features spectral tunability from UV to hard X-rays. Moreover, advantage of particular importance is a very high repetition rate of HHG which is given by the repetition rate of the driving laser only and can be easily as much as few $\mathrm{kHz}$ (Schultze et al., 2007)!

It has been shown that high-order harmonic pulse comprises train of attosecond pulses (Papadogiannis et al., 1999). This great advantage constitutes a stimulus for further development of high-order harmonic sources, especially of the techniques leading to generation of single attosecond pulses. Nowadays, well explored and most frequently deployed are:

- usage of very short IR laser pulses ( < 5 fs) (Christov et al., 1997; Baltuska et al., 2003),

- a technique called polarization gating (Sola et al., 2006).

The details of the aforementioned techniques will not be discussed in detail here; however, it is worth noting that the intension of improvement of high-order harmonic sources has become a boost for laser technology progress leading to development of laser systems emitting pulses with duration in the range of single optical cycle $(\sim 3.3 \mathrm{fs}$ at $\sim 810 \mathrm{~nm}$ central wavelength) and shifting the laser pulse central wavelength to the mid-infrared spectral range (MIR) in around 2-3 $\mu \mathrm{m}$. Besides, the lasers' repetition rates have been significantly increased typically to a few $\mathrm{kHz}$ (and energy $\sim \mathrm{mJ}$ per pulse; e.g. Schultze et al., 2007). Another recent achievement of particular interest is carrier-envelope absolute phase stabilization (CEP).

State-of-the-art HHG sources require not only development of the high-harmonic source itself but also sophisticated metrology techniques and methods for characterization of femtosecond and attosecond pulses (Véniard et al., 1996; Drescher et al., 2002; Kienberger et al., 2002; Mairesse et al., 2005; Itatani et al., 2002; Sansone et al., 2008).

Due to unusual combination of all properties that high-order harmonics feature, they immediately found vast number of unprecedented applications. For example, a number of

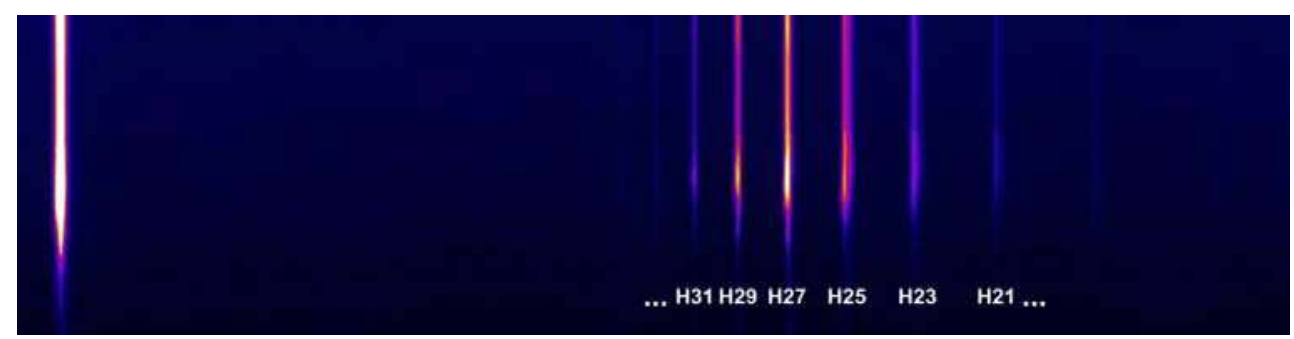

Fig. 2. Typical spectrum of high-order harmonics (conversion medium: argon; Jakubczak a)). 
experimental results have been recently published related to time-resolved investigation of atomic processes. For instance manipulation of drift energy of photoelectron wave packets (so called "steering of wave packets") and their imaging (e.g. Kienberger et al., 2007), measurement of relaxation and lifetime dynamics in an atom by the direct measurement in time domain with attosecond resolution (e.g. Baltuska et al., 2003; Kienberger et al., 2002) in contrary to thus far frequency-domain measurements of transition linewidths (Becker \& Shirley, 1996), spectroscopy of bound electron dynamics in atoms and molecules (Hentschel et al., 2001), observation of interference of coherent electron wave packets (Remetter et al., 2006), probing molecular dynamics (Niikura et al., 2002) and real-time tomography of molecular orbitals (Itatani et al., 2004).

Moreover, novel and very promising schemes for HHG have been recently demonstrated, e.g., generation of harmonics during reflection of super intense ultrashort IR laser pulses $\left(\mathrm{I}>1017 \mathrm{~W} / \mathrm{cm}^{2}\right)$ from plasma mirror oscillating at relativistic velocities on the surface of a solid state target (Quéré et al., 2006), or generation of HHG from interaction of IR femtosecond laser pulses with molecules $\left(\mathrm{N}_{2}, \mathrm{H}^{2+}\right.$; Lorin et al., 2008).

\section{Physical mechanisms of high-order harmonic generation}

If material is subjected to a strong electric field, nonlinear polarization of the material is induced. The magnitude of the arisen polarization strongly depends on the intensity of the incident radiation. At moderate and low intensity values the external electric field does not influence significantly the electronic structure of the irradiated atoms. The potential barriers can be just slightly modified and Stark effect can be observed. To great probability the atoms remain in their ground state and extension of their ground state wave function is of the order of Bohr radius $\left(5.2917 \cdot 10^{-11} \mathrm{~m}\right)$. All nonlinear phenomena taking place in this regime are well described by the perturbation theory. Thus it is referred as the perturbative regime of nonlinear optics. Comprehensive discussion on phenomena and related theory in the perturbative regime can be found e.g. in Boyd, 2003. Some of nonlinear optical phenomena in this regime are:

- harmonic radiation generation (second, third, etc.),

- optical parametric amplification,

- optical rectification,

- stimulated Raman scattering,

- $\quad$ self-phase modulation,

- $\quad$ self-focusing.

However, when the electric field strength of the incident radiation is comparable to (or higher than) atomic electric field strength $\left(5.142 \cdot 10^{11} \mathrm{~V} / \mathrm{m}\right.$; Brabec \& Krausz, 2000) then the potential barriers are strongly modified. With high probability the electrons from the most-outer atomic shells may be liberated either through the tunnel ionization or the abovebarrier ionization (depending on the external field strength; see Fig. 3 and Fig. 4). Subsequently, if the field is linearly polarized electron wave packets will start oscillatory motion. The amplitude of oscillations exceeds Bohr radius and cycle-averaged kinetic energy of electron wave packet surpasses binding energy (Brabec \& Krausz, 2000).

Range of intensities implying these phenomena defines the strong field nonlinear optics regime. In contrary to the perturbative regime, here, the nonlinear response of the polarization of the medium is affected by the ionization process. The nonlinear treatment can be only applied 


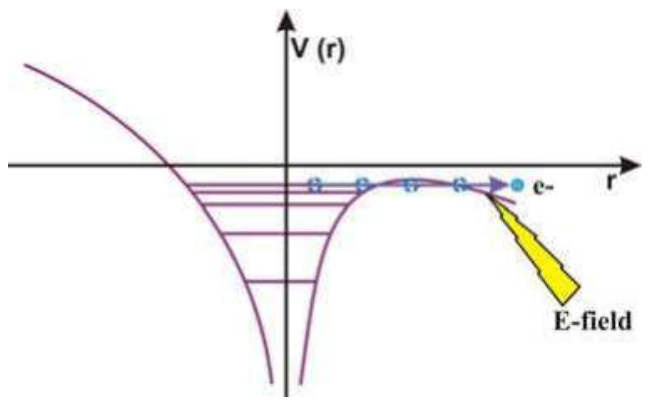

Fig. 3. Tunnel ionization. The atomic potential affected by the external electric field whose the field strength is comparable to the atomic fields. It is plausible that the electrons from the most-outer atomic will be unbound. This transition is often referred as optical field ionization $(\mathrm{OFI})$.

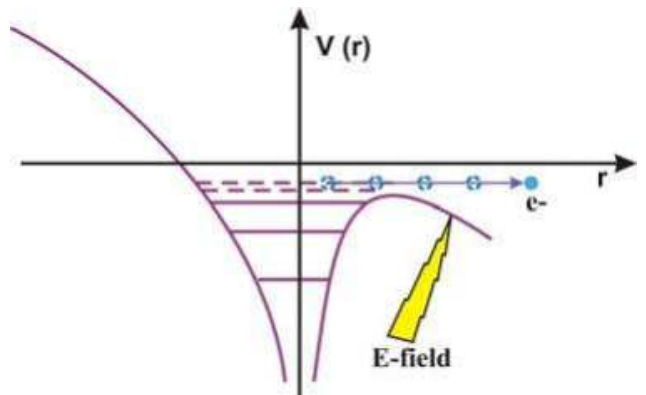

Fig. 4. In this case, the applied electric field is higher than the atomic field strength. The atomic potential barrier is suppressed and electrons from most-outer shells are liberated through above barrier ionization.

to an electron which is in very close vicinity of a parent ion. As soon as it is released by optical field its response is linear to the electric field and may be treated by classical laws of motion (Corkum, 1989; Corkum, 1993).

Very interesting phenomena are present in the intermediate range of parameters, in the so called intermediate regime, i.e. between the perturbative and the strong field regimes. They include long-distance self-channeling when nonlinear Kerr effect causes beam focusing, on the one hand, and free electrons cause its defocusing, on the other. This interplay leads to the channeling of the propagating intense pulse (even at distances as long as a few meters). Another interesting phenomenon in this regime is multiphoton ionization, where the total amount of absorbed energy exceeds the ionization potential (Fig. 5).

When electric field strengths are even higher, the nonlinearities become stronger. Electric field is able to optically liberate electrons from inner shells of the atom and the wiggling energy of an electron is comparable with its rest energy $\mathrm{mc}^{2}$. This is a launch of relativistic regime.

Publications of crucial importance related to the intermediate to strong-field nonlinear optics regimes were made by Keldysh (Keldysh, 1965) and Ammonsov, Delone and Krainov (Ammosov et al., 1986). Keldysh defined a parameter, which was later named after him that allows determining whether tunneling or multiphoton process is dominant for particular experimental conditions. It reads: 


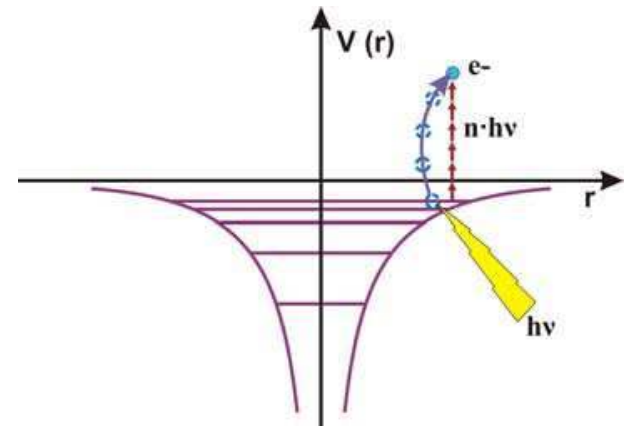

Fig. 5. Multiphoton ionization process: n-photons are absorbed. The total energy of absorbed photons ( $\mathrm{n}$ *hv; $\mathrm{n}$ - number of absorbed photons, $\mathrm{h}$ - Planck's constant, $\mathrm{v}$ - light frequency) exceeds ionization potential.

$$
\gamma=\sqrt{\frac{I_{p}}{2 \cdot U_{p}}}
$$

Where:

$\mathrm{I}_{\mathrm{p}}$ - is ionization potential of a nonlinear medium,

$U_{p}$ - is ponderomotive potential, which is cycle-averaged quivering energy of an electron in the external laser field. It is defined as:

$$
U_{p}=\frac{e^{2} \cdot E_{0}^{2}}{4 m_{e} \cdot \omega^{2}}
$$

Where:

e - stands for charge of electron,

$\mathrm{m}_{\mathrm{e}}$ - is mass of electron,

$E_{0}$ - external field amplitude oscillating at frequency $\omega$.

Substitution of constants leads to simplified relation:

$$
U_{p}[e V]=0.97 \cdot 10^{-13} I\left[W / \mathrm{cm}^{2}\right] \lambda^{2}[\mu m]
$$

The laser field amplitude can be estimated from relation:

$$
E_{0}{ }^{2}[\mathrm{~V} / \mathrm{cm}]=\frac{I\left[W / \mathrm{cm}^{2}\right]}{\frac{1}{2} Z_{0}}
$$

Where:

$\mathrm{I}$ - is laser intensity $\left[\mathrm{V} / \mathrm{cm}^{2}\right]$,

$\mathrm{Z}_{0}$ - is vacuum impedance.

$$
Z_{0}=\sqrt{\mu_{0} \varepsilon_{0}}=377[\mathrm{~V} / \mathrm{A}]
$$

Where:

$\mu_{0}$ - is vacuum permeability, $\mu_{0}=1.26 \cdot 10^{-6}[\mathrm{H} / \mathrm{m}]$, 
$\varepsilon_{0}$ - is vacuum permittivity, $\varepsilon_{0}=8.85 \cdot 10^{-12}[\mathrm{~F} / \mathrm{m}]$.

If $\gamma \gg>1$ multiphoton ionization dominates. However, if $\gamma<<1$ tunneling ionization takes over. By these simple formulas it is possible to divide regimes of nonlinear optics in intensity domain as depicted in Fig. 6.

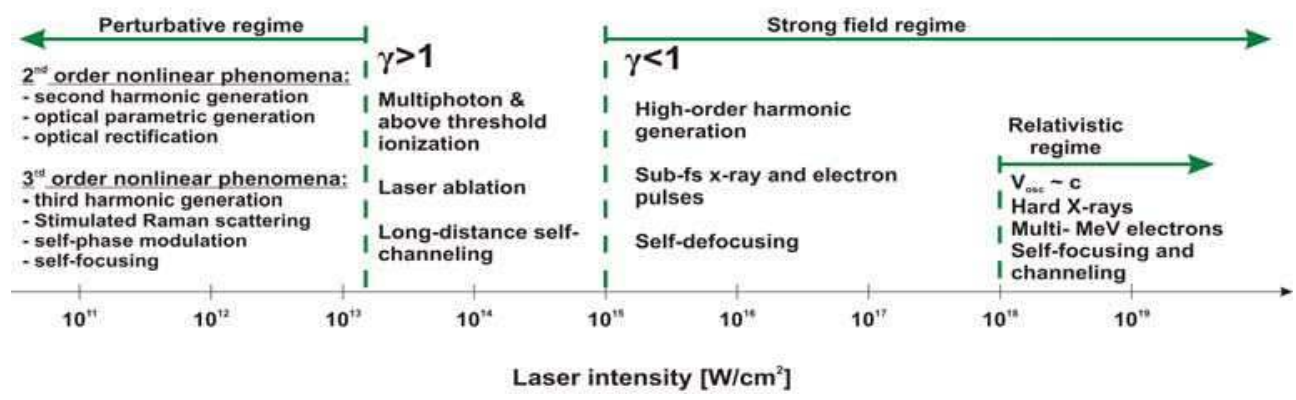

Fig. 6. Regimes of nonlinear optics.

Ionization rates are of core importance when discussing interaction of the intense laser pulses with matter. Their estimations were performed within quastistatic field approximation. The first approach was proposed by Keldysh. The second was developed by Ammonsov, Delone and Krainov (also known as ADK theory named after the acronyms of the names). Additionally, the ionization rate calculation could be performed by an exact numerical solution of the time-dependent Schrödinger equation. The Keldysh theory possesses a source of a discrepancy between the other theories which comes from the fact that it neglects Coulomb potential in an atom. The difference leads to lower ionization rates compared to other approaches (e.g. in case of $\mathrm{He}$ and $\mathrm{H}$ by about 1-2 orders of magnitude) (Brabec \& Krausz, 2000). The discrepancies between theories increase with the electric field strength. For example, for He there is no difference between ionization rates obtained from ADK theory and solution of the time-dependent Schrödinger equation as long as the field strength does not exceed 0.2 atomic unit.

\section{High-order harmonic generation in gaseous media}

\subsection{Microscopic analysis}

High-order harmonic generation process takes place when linearly polarized ultrashort laser pulses of intensity of a few times $10^{13} \mathrm{~W} / \mathrm{cm}^{2}$ to $<10^{16} \mathrm{~W} / \mathrm{cm}^{2}$, and time duration from picoseconds to a few femtoseconds (Pfeifer et al., 2006), are applied to a nonlinear medium (atoms, atom clusters, molecules and plasmas).

HHG process can be understood using semi-classical three-step model (Corkum, 1993):

Step I - Ionization. When an atom is exposed to external electric field, the potential of the atom is modified by a factor of $e \vec{E}(t) \vec{r}$. Then, the resulting potential is equal to:

$$
V(\vec{r}, t)=-\frac{e^{2}}{4 \pi \varepsilon_{0} r}+e \vec{E}(t) \vec{r}
$$

With increasing strength of the external field $\vec{E}(t)$ the probability of tunnel-ionization by the low-frequency laser field of most-outer-shell electrons increases significantly (the natural potential of atom is being cancelled). 
Step II - Propagation. When an electron wave function undergoes tunnel-ionization from its parent atom the free electron wave packet is affected primarily by the external electric field (and not by the field of the parent atom) and is accelerated by this field. When the laser field reverses its sign, the electrons slow down and are re-accelerated back towards the atom. The free electron motion can be described by laws of classical physics (Pfeifer et al., 2006):

$$
v(t)=\int_{0}^{t}-\frac{e}{m} E\left(t^{\prime}\right) d t^{\prime}+v_{0}=-\frac{E_{0} e}{m \omega} \sin (\omega t)+v_{0}
$$

where: $\mathrm{v}(\mathrm{t})$ - is instant velocity of an electron, $\mathrm{v}_{0}$ - is electron drift velocity.

If we consider an initially bound electron $(x=0)$, with zero drift velocity, its velocity can be described by (Pfeifer et al., 2006):

$$
v(t)=\int_{0}^{t}-\frac{e}{m} E\left(t^{\prime}\right) d t^{\prime}+v_{0}=-\frac{E_{0} e}{m \omega}[\sin (\omega t+\varphi)-\sin (\varphi)]
$$

and its position (Pfeifer et al., 2006):

$$
x(t)=\int_{0}^{t} v\left(t^{\prime}\right) d t^{\prime}=\frac{E_{0} e}{m \omega^{2}}[\cos (\omega t+\varphi)-\cos (\varphi)]+\sin (\varphi) t
$$

Where: $\varphi$ is phase of electric field at which atom is ionized (often referred as: "electron is born").

Dependence of the electrons paths as a function of time, where the electric field phase is a parameter, reveals that just a fraction of the electrons are probable to return to the parent ion and re-collide (contributing to emission of radiation). The problem is addressed in more detail in Pfeifer et al., 2006. This is the reason of the optimization of the electric field phase.

Step III - Recombination. After re-acceleration of the electron wave packet towards the ion it is plausible that electron will collide with the ion and recombine. The excess of kinetic energy is transferred to the momentum of the emitted photon. The electrons which recombine with ions will emit harmonic radiation with energy of spectral lines defined as follows:

$$
\hbar v=E_{k i n}+I_{p}
$$

Where $E_{\text {kin }}$ - is kinetic energy of an electron acquired by absorption of n-photons of the driving field, $I_{p}$ - is ionization potential corresponding to the shell from which the electron has been ripped off by the field.

There exists a limit on the maximum emitted energy. It is given by maximum kinetic energy that electron gains during acceleration. It was claimed that the path and kinetic energy of the electron is controlled by the phase of the electric field (Eq. 7 - Eq. 9). If phase is $\sim 18^{\circ}$ the kinetic energy of electron is maximized and its value is $\sim 3.17$. Up (Pfeifer et al., 2006) ${ }^{1}$. Thus the energy of the highest harmonic order is given by:

$$
\hbar v=3.17 U_{p}+I_{p}
$$

\footnotetext{
${ }^{1} 18^{\circ}=\sim 314 \mathrm{mrad}$.
} 


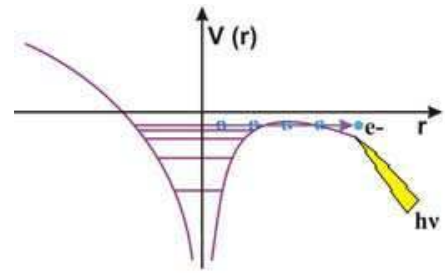

Step 1: Ionization

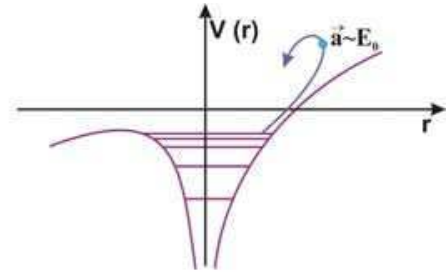

Step 2: Acceleration

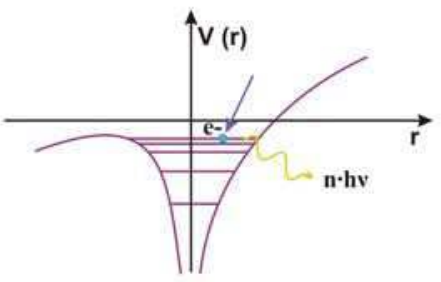

Step 3: Recombination

Fig. 7. Summary of the 3-step model of HHG. The first step is tunnel-ionization of an atom. Next, an electron is driven away from its parent ion in the external electric field of an intense laser pulse. When the oscillating laser field reverses its sign the particle is re-accelerated back towards the atom and, finally, recombines. The last step leads to emission of a photon.

The HHG process can be also explained in the formalism of quantum mechanics (Itatani et al., 2004). The returning electron wave packet overlaps remaining portion of initial wave function. The coherent addition of the two wave functions induces a dipole as asymmetric displacement of the electron wave packet. The dipole oscillates as continuum wave function (of the free electron packet) propagates. The oscillating dipole is a source of harmonic radiation and harmonic spectrum is given by Fourier transform of dipole acceleration. Instantaneous frequency of the dipole is determined by kinetic energy of the recombining electrons (i.e. electron wave packet and emitted photons are linked by the energy conservation: $\hbar v=E_{k}$, where the ionization potential is omitted due to the fact that $E_{k}$ of electrons is "seen" by bound-state electron wave function, compare to Eq. 11). It is worth noting that the electron wave packet and the emitted photons are mutually coherent.

It is important to point the influence of tunnel-ionization process on HHG. The ionization rate increases with the amplitude of the electric field (Keldysh, 1965; Ammosov et al., 1986) leading to generation of free electrons what results in their increasingly stronger contribution to HHG. Most energetic electrons are produced at $18^{\circ}$-phase of the electric field. On the other hand, multiphoton ionization produces constant number of electrons depending only on the intensity of the laser pulses and not on the field phase. From cut-off law (Eq. 11) we also know that energy of photons may be increased by increasing the ponderomotive potential $\mathrm{U}_{\mathrm{p}}$ (when $\gamma<<1$ ), thus shifting more into the strong-field regime.

\subsection{Macroscopic analysis - phase-matching considerations}

In most generic sense HHG process analysis is divided into two logical constituents: microand macroscopic. The three-step model deals only with atomic-scale phenomena leading to emission of a photon, thus, this part of the process is assigned to microscopic analysis of 
HHG. However, there are also macroscopic issues to be concerned. The crucial problem in this sense for HHG process is a phase-matching of the two propagating beams: XUV from HHG and of the driving laser. If we consider mismatch between $\mathrm{m}^{\text {th }}$ harmonic order and driving laser field oscillating at fundamental frequency $\omega_{\mathrm{f}}$ the wave vector of the mismatch could be written as (Pfeifer et al., 2006):

$$
\Delta k=m k\left(\omega_{f}\right)-k\left(m \omega_{f}\right)
$$

In general there are three major components of total $\Delta k$ that may be written as:

$$
\Delta k=\Delta k_{\text {natural }}+\Delta k_{\text {plasma }}+\Delta k_{\text {foc }}
$$

Refractive index is a function of frequency. Since the phase velocity of radiation at given wavelength depends on refractive index, in general, radiation of different wavelengths propagates with different velocities what leads to de-phasing of particular spectral components. This input to total phase mismatch is called natural dispersion.

Because only little fraction of free electrons that had been generated by the laser field recombines with parent ion free-electron clouds are created. These free electrons give raise to additional component of refractive index (Brabec \& Krausz, 2000):

$$
\begin{gathered}
n_{\text {plasma }}=\sqrt{1-\frac{N_{e}}{N_{c}\left(\omega_{f}\right)}} \\
N_{c}(\omega)=\frac{\varepsilon_{0} m_{e} \omega^{2}}{e^{2}}
\end{gathered}
$$

Where:

$\mathrm{N}_{\mathrm{e}}$ - is free-electron density,

$\mathrm{N}_{\mathrm{c}}$ - is critical plasma density.

This leads to phase mismatch wave vector component (Pfeifer et al., 2006):

$$
\Delta k_{\text {plasma }}=m k_{\text {plasma }}\left(\omega_{f}\right)-k_{\text {plasma }}\left(m \omega_{f}\right)=\frac{\omega_{p}^{2}\left(1-m^{2}\right)}{2 m c \omega_{f}}
$$

HHG requires peak intensities in range of $10^{13}-10^{16} \mathrm{~W} / \mathrm{cm}^{2}$. In order to obtain such high intensities one has to focus driving laser beam. However, focusing involves phase-shift of the driving field along beam propagation direction ' $z$ ' (so called Gouy phase shift; Jaeglé, 2006):

$$
\varphi_{\text {geo }}(z)=-\arctan \left(\frac{2 z}{b}\right)
$$

Where "b" is a confocal parameter defined as follows (Jaeglé, 2006):

$$
b=\frac{2 \pi a^{2}}{\lambda}
$$

Where "a" is beam radius in the focal spot. 
Gouy phase shift leads to the phase mismatch wave vector component (Pfeifer et al., 2006):

$$
\Delta k_{f o c}=m k_{f o c}\left(\omega_{f}\right)-k_{f o c}\left(m \omega_{f}\right)=\frac{2(m-1)}{b}
$$

If the HHG process takes place in a hollow fiber the $\Delta k_{f o c}$ component is replaced by:

$$
\Delta k_{\text {cap }}=m k_{\text {cap }}\left(\omega_{f}\right)-k_{c a p}\left(m \omega_{f}\right)=\frac{u_{n l}{ }^{2} c\left(1-m^{2}\right)}{2 m d^{2} \omega_{f}}
$$

Where:

$\mathrm{u}_{\mathrm{nl}}$ - is $\mathrm{l}^{\text {th }}$ zero of Bessel function, and

$\mathrm{d}$ - is capillary inner radius.

There is also a component to phase mismatch originating from the fact that when the driving field pulse propagates in a gas it is defocused by the free electrons density gradient what leads to drop of intensity. Since the dipole moment is roughly linearly proportional to $-U_{p} / \omega_{f}$ the intensity drop implies de-phasing. However, this contribution is negligible compared to the de-phasing due to focusing and free-electrons generated index of refraction.

Phase-matching condition can be fulfilled by:

- tuning gas density (modification of gas density leads to modification of the index of refraction),

- changing position of the focus with respect to the gas resulting in minimization of Gouy phase-shift influence (gradient of function defined by Eq. 17 is highest at the beam waist),

- in the hollow-fiber geometry modification of the fiber parameters can develop perfect phase-matching,

- $\quad$ free electron density may be controlled by intensity and time duration of laser pulses.

\subsection{Generic properties of high-order harmonics spectrum}

An important feature of the harmonic spectrum is its universal shape. As already mentioned there is a spectral region of roughly equally intense spectral lines, so called plateau. The plateau is preceded by increase of spectral lines intensity in longer wavelengths which is subsequently followed by abrupt intensity drop-off. The short-wavelength part of spectrum extends to the limit defined by cut-off law. From this formula (Eq. 11) one can infer how to extend the wavelength range of HHG, e.g., by increasing ionization potential (proper choose of conversion medium or by working with ions for subsequent shells have higher ionization potential). Another way of doing so is by raising intensity or increasing wavelength of driving laser field (revoke Eq. 2 or Eq. 3).

It has been shown that intensity of $\mathrm{m}^{\text {th }}$ harmonic order is proportional to square of phasematching factor ( $\mathrm{m}^{\text {th }} \propto|\mathrm{Fq}|^{2}$; Jaeglé, 2006). This factor rapidly decreases with $m$ leading to drop of intensity of spectral lines in the long-wavelength part of harmonic spectrum.

Additionally, in the plateau region the scaling law has been observed (Jaeglé, 2006):

$$
I_{m} \propto b^{3} \cdot \Delta t
$$

Where, $\mathrm{I}_{\mathrm{m}}$ - is intensity of $\mathrm{m}^{\text {th }}$ harmonic order, $\mathrm{b}$ is confocal parameter, $\Delta \mathrm{t}$ is driving laser pulse duration. The dependence on $\mathrm{b}^{3}$ comes from the fact that total number of photons is 
spatially integrated. The dependence on duration of the pulse comes from temporal integration of the HHG process.

In most common scheme of HHG when only one-wavelength driving laser field is used (in gaseous media) the HHG spectra contain only odd harmonic orders. This could be understood by formalism presented, e.g., in Boyd, 2003. This approach originates from perturbative theory of description of nonlinear optical phenomena and is followed here after Boyd.

When low-strength external field $\vec{E}(t)\left(\ll<10^{9} \mathrm{~W} / \mathrm{cm}^{2}\right)$ interacts with matter the material polarization $\vec{P}(t)$ (or dipole moment per unit volume) is responding to excitation in linear fashion to the applied field. This relation is linked by linear susceptibility and reads:

$$
\vec{P}(t)=\chi^{(1)} \vec{E}(t)
$$

However, if the field increases the nonlinear response of the harmonic oscillator appears. If the field is not too strong (for intensities smaller than $\sim 10^{14} \mathrm{~W} / \mathrm{cm}^{2}$ ) $\vec{P}(t)$ can be expanded in Taylor series:

$$
\vec{P}(t)(t)=\chi^{(1)} \vec{E}(t)+\chi^{(2)} \vec{E}^{2}(t)+\chi^{(3)} \vec{E}^{3}(t)+\chi^{(4)} \vec{E}^{4}(t)+\ldots
$$

If any arbitrary medium features e.g. third order nonlinearity $\left(\chi^{(3)}\right.$ does not vanish) the medium polarization is capable of being a source of nonlinearities of the third order (e.g. third-harmonic generation, nonlinear index of refraction and secondary phenomena having their origin in the dependence of $n=f(I)$, etc.). In general, there is a strong dependence between type of symmetries of media and their nonlinear properties. For instance, if a medium is centrosymmetric all its even-order susceptibilities vanish and thus those media are not able to give rise to any of even-order nonlinear phenomena. This is also the case of gasses (as well as e.g. liquids and amorphous solids). Since gases display inversion symmetry it is possible to obtain odd-order HHG only.

\subsection{HHG geometries involving gaseous media}

The most common experimental setup of HHG comprises (apart from laser system and diagnostic apparatus) a gas puff target (L'Huillier \& Balcou, 1993). Such a target is basically a gas valve injecting a portion of a gas at desired pressure. The valve is repetitively open and laser pulses interact in proximity of valve exhaust. Typical repetition rate of the gas puffs is $<100 \mathrm{~Hz}$. The valves may have either circular symmetry or could be elongated. Elongated valves provide higher XUV beam outputs but limiting factor is re-absorption in a gas thus long valves are used for wavelengths $>\sim 20 \mathrm{~nm}$ (also because of the longer coherence length for longer wavelengths). On the other hand, circular (e.g. $0.5 \mathrm{~mm}$ diameter) gas puff valves are used in shorter-wavelength HHG.

High-order harmonics are also generated in gas cells. A gas cell is a simple container filled with a noble gas at moderate pressure (few tens of mbar). Arrangements with gas cells are very comfortable to work with because, compared to the gas puff targets, there are fewer parameters to optimize to obtain phase matching. In this case the phase matching is obtained by tuning only longitudinal position of a cell and gas pressure in it. For gas cells 
can be as long as desired (due to technical ease of construction compared to gas puff valves) this setup is also favorable when maximization of interaction length is wanted.

Another possible geometry of HHG involves hollow fibers filled with a conversion gas (Rundquist et al., 1998). In such a fiber laser pulses are propagating even meters long resulting in efficient transfer of driving field energy into XUV beam. This geometry is also popular due to ease of control of phase-matching by the fiber parameters (Eq. 20).

\section{High-order harmonic generation by molecules}

Essentially, the physical mechanism of HHG in molecules is the same as in atomic gases (see section 3) and can be understood by the same 3-step model. However, orbital structure of molecules and thus the description of the mechanism of HHG in this case is significantly different from situation when atomic conversion media are involved.

There have been many molecules proven to be capable of HHG:

- $\quad \mathrm{N}_{2}$ (Itatani et al., 2004; Sakai \& Miyazaki, 1995),

- $\quad \mathrm{CS}_{2}$, hexane, $\mathrm{N}_{2}$ (Velotta et al., 2001),

- $\mathrm{O}_{2}, \mathrm{CO}_{2}$ and $\mathrm{N}_{2}$ (Kanai et al., 2007).

A very interesting feature of HHG in molecules is that signal yield of particular harmonic order depends on laser light polarization ellipticity and its orientation with the respect to the direction of molecular axis. In general, the signal yield is highest for linearly polarized light perpendicularly oriented to the axis of the molecule (Kanai et al., 2007).

The fact that yield of HHG depends on orientation of the molecule with the respect to light polarization direction suggests that the HHG process (especially in molecules) is strongly affected by the shape of orbitals of the molecule. This idea has been motivation to Itatani et al., 2004. Finding the relation between orientation of the molecule and spectral intensities of high-order harmonics they succeeded to perform inverse calculation and obtained tomographic reconstruction of the most-outer orbital of $\mathrm{N}_{2}$ molecule. Since these orbitals are responsible for chemical properties of the molecules the results have great impact on the state of our knowledge. Such direct measurement of orbitals is a first step to the "molecular movie" showing, e.g., time-resolved process of creation of chemical bonds.

Another very interesting feature of HHG from molecules was unveiled during experiments in which the influence of molecular structure complexity on HHG efficiency was investigated. It turned out that the increasing complexity of the molecule is unfavorable for efficient HHG. When dissociating pre-pulse was applied the HHG yields were higher compared to case with unaffected molecules (Velotta et al., 2001; Hay et al., 2002). It has been inferred (Jaeglé, 2006) that the origin of the higher conversion efficiency in atomic media relies in fact that dipole phase depends on the angle between molecular axis and the pump laser polarization. An additional de-phasing mechanism would exist between the emitters in randomly aligned molecular media and it could imply worse phase matching compared with the monomers (Hay et al., 2002).

\section{High-order harmonic generation from solid targets}

HHG process can also take place at the interface between vacuum and solid targets. The physical process leading to HHG is different here and is explained in terms of resonant absorption. Let us now introduce reference system presented in Fig. 8. 


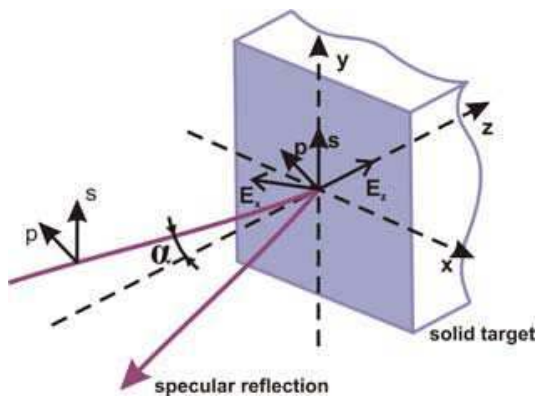

Fig. 8. HHG from solid target surface geometry.

From electromagnetic analysis of reflection of radiation at an interface between two media it is known that in case of specular reflection at oblique angles $\left(\alpha>0^{\circ}\right)$ "s" polarization is reflected according to Snell's law, or, alternatively, this polarization, does not propagate in z direction (due to the boundary conditions). On the other hand, "p" polarization can propagate in $\mathrm{z}$ direction into the medium. When a very intense laser pulse impinges upon the target the plasma is created. In such a plasma light propagates to the layer of freeelectron density equal to plasma critical density (Eq. 24). The laser radiation cannot propagate any farther, but, instead, the free-electron plasma wave (plasmon) is induced by "p" polarized light at frequency equal light frequency (resonant absorption) and directionality of propagation along $\mathrm{z}$ axis.

$$
N_{c}=\frac{4 \pi^{2} c^{2} \varepsilon_{0} m_{e}}{e^{2} \lambda_{L}^{2}} \approx \frac{1.1 \cdot 10^{21}}{\lambda_{L}^{2}[\mu m]}\left[\mathrm{cm}^{-3}\right]
$$

When laser light at frequency $\omega_{\mathrm{L}}$ mixes with the induced plasmon at frequency $\omega_{\mathrm{p}}$, the light at frequency of $2 \omega_{\mathrm{L}}$ is generated. The $2 \omega_{\mathrm{L}}$-electromagnetic wave may propagate further into plasma since plasma critical density for $2 \omega_{\mathrm{L}}$ is higher $\left(4 \mathrm{~N}_{\mathrm{c}}\right)$. Then, light at $2 \omega_{\mathrm{L}}$ mixes with that at $\omega_{\mathrm{p}}$ generating $3 \omega_{\mathrm{L}}$ component. This frequency radiation can propagate until it reaches density of $9 \mathrm{~N}_{\mathrm{c}}$, and so on (up to some upper-limit density $\mathrm{N}_{\mathrm{u}}$ ). This cascaded phenomenon leads to generation of harmonics of the order limited by plasma frequency and its maximum value is given by (Jaeglé, 2006):

$$
q_{\max } \approx \frac{N_{u}}{N_{c}}
$$

The approximate relation for $\mathrm{N}_{\mathrm{u}}$ is given by (Jaeglé, 2006):

$$
N_{u}=\frac{E_{0}^{2}}{8 \pi T_{b}}
$$

$\mathrm{T}_{\mathrm{b}}$ is background temperature (Carman et al., 1981).

Very important parameter is $I \cdot \lambda^{2}\left[\mu \mathrm{m}^{2} \mathrm{~W} / \mathrm{cm}^{2}\right]$ product. For $\lambda=1 \mu \mathrm{m}$ and intensities below $10^{18} \mathrm{~W} / \mathrm{cm}^{2}$ Lorentz force can be approximated only by component coming from electric field, i.e. by $\mathrm{e} \cdot \mathrm{E}_{\mathrm{z}}$. However, if intensity becomes comparable to $10^{18} \mathrm{~W} / \mathrm{cm}^{2}$ the relativistic effects bring increasingly significant input to Lorentz force from magnetic field component. 
For laser pulse intensities below the mentioned value (or for $\mathrm{I}^{2}<10^{18} \mu \mathrm{m}^{2} \mathrm{~W} / \mathrm{cm}^{2}$ ) the only polarization that may induce harmonic generation is " $p$ " polarization. However, above this value HHG may take place due to nonlinear mixing between longitudinal and transverse oscillations resulting in possibility of HHG from "s" polarized light (Jaeglé, 2006).

Another way of describing of HHG from solid-vacuum interface involves an oscillating plasma mirror. It could be shown by mathematical analysis that light reflected at critical surface oscillating at relativistic velocities contains harmonics of the incident beam (Jaeglé, 2006). This approach; however, will not be discussed in more detail here. Instead, some general properties of HHG from solids shall be discussed. Probably the most interesting feature of HHG from solids is that all-order harmonic radiation is obtained. Additionally, it has been observed that the emission solid angle changes with the intensity of driving laser field (Jaeglé, 2006). In general the emission cone is larger compared to the laser beam. When $\mathrm{I} \lambda^{2}$ reaches values of $10^{15}-10^{16} \mu \mathrm{m}^{2} \mathrm{~W} / \mathrm{cm}^{2}$ the emission solid angle strongly increases; for values above $10^{17} \mu \mathrm{m}^{2} \mathrm{~W} / \mathrm{cm}^{2}$ it is found to be isotropic (no angular distribution of HHG has been found - Jaeglé, 2006). Moreover, HHG efficiency and signal yield from solids drop above $10^{16} \mathrm{\mu m}^{2} \mathrm{~W} / \mathrm{cm}^{2}$. The two phenomena are attributed to the transition from specular reflection regime of HHG to diffusion reflection regime.

The key parameters of HHG from solid-vacuum interfaces sources are:

- Intensity of the driving field (or more exactly the product of $I \lambda^{2}$ ), which is basically the only limiting factor to the order of generated harmonics,

- $\quad$ angle of incidence of the driving field upon the target,

- $\quad$ contrast of the driving laser pulse.

Nowadays, this type of HHG source is drawing lots of attention in the scientific world. This is due to huge potential capabilities of this type of source (e.g. odd and even harmonic orders generation and their number limited only by available intensity of the driving laser fields, conversion efficiency increasing with $\mathrm{I}^{2}$ ). For more detailed discussion on HHG from solids see, e.g., (Carman et al., 1981; Bezzerides et al., 1982; Dromey et al., 2006; Balcou et al., 2006; Tarasevitch et al., 2007; Quéré et al., 2008; Thaury et al., 2007).

\section{Applications of high-order harmonic sources}

High-order harmonics, due to their high repetition rate operation, tunability and high coherence degree, have already found a number of interesting applications. For instance, they are used in material sciences, life sciences and detection technology. As an example high-order harmonics together with femtosecond NIR beam were used to efficiently modify tribological properties of materials. The results from these experiments are presented in the sub-section 6.1. High-order harmonics are also very practical in metrology of multilayer (ML) optics (sub-section 6.2).

\subsection{Materials surface processing}

Recently, a new method for materials processing suitable for efficient machining of transparent materials has been demonstrated (Mocek et al., 2009). The technique utilizes simultaneous interaction of NIR femtosecond laser pulses generated by $\mathrm{Ti}: \mathrm{Al}_{2} \mathrm{O}_{3}$ laser system $\left(\Delta \mathrm{t}=32 \mathrm{fs}, \mathrm{E}=2.8 \mathrm{~mJ}, \lambda_{\mathrm{c}}=820 \mathrm{~nm}\right)$ and the second harmonic, combined with extreme ultraviolet (XUV) high-order harmonics with the strongest spectral line at $21.6 \mathrm{~nm}$. 


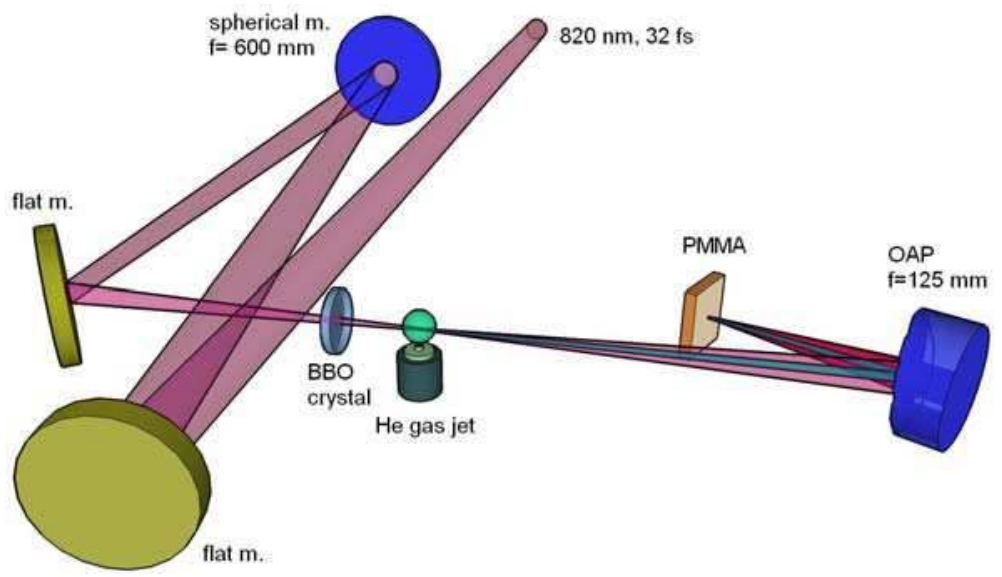

Fig. 9. Schematic of the experimental setup for surface modification by dual action of XUV and Vis-NIR ultrashort pulses (Mocek et al., 2009).

The experimental setup is shown in Fig. 9. For strong HHG a two-color laser field, consisting of fundamental and second harmonic $(\mathrm{SH})$ of a femtosecond laser pulse, was applied to a gas jet of He (Kim et al., 2005). Femtosecond laser pulses at $820 \mathrm{~nm}$ with an energy of $2.8 \mathrm{~mJ}$ and pulse duration of $32 \mathrm{fs}$ were focused by a spherical mirror $(f=600 \mathrm{~mm})$ into a He gas jet. For SH generation, a $200-\mu \mathrm{m}$-thick beta-barium borate (BBO) crystal was placed between the focusing mirror and gas jet so that, after the BBO crystal, the laser field consisted of both the $\mathrm{SH}$ and the residual fundamental laser fields. For the optimum $\mathrm{SH}$ conversion the $\mathrm{BBO}$ crystal was placed $\sim 400 \mathrm{~mm}$ from the focusing mirror and the energy conversion efficiency was about $27 \%$. A gas jet with a slit nozzle of $0.5 \mathrm{~mm}$ width and length of $6 \mathrm{~mm}$ was used (Kim et al., 2008). The gas pressure in the interaction region was 150 Torr ( 0.2 bar). Generated HHG were first characterized using a flat-field soft X-ray spectrometer equipped with a back-illuminated X-ray charge coupled device. Optimization of the two-color HHG source was performed by selecting the gas jet position while controlling the relative phase between the two fields. The strongest harmonic at the $38^{\text {th }}$ order (at $21.6 \mathrm{~nm}$ ) reached energy of $\sim 50 \mathrm{~nJ}$.

Subsequently, the spectrometer was replaced with a 1-inch diameter off-axis paraboloidal mirror (OAP, $f=125 \mathrm{~mm}$ at $\left.13^{\circ}\right)$ with a Mo:Si multilayer coating $(R=30 \%$ at $21 \mathrm{~nm})$ placed $245 \mathrm{~mm}$ from the HHG source. The sample (500-nm thin layer of PMMA spin-coated on a $315 \mu \mathrm{m}$ thick silicon substrate) was positioned $125 \mathrm{~mm}$ from the OAP, perpendicularly to the incident beam. The measured reflectivity of the OAP in the optical region was $37 \%$. The measured diameter (FWHM) of the HHG beam incident on the OAP was $280 \mu \mathrm{m}$ while the diameter of the fundamental and $\mathrm{SH}$ laser beams was $\sim 4 \mathrm{~mm}$. The morphology of irradiated target surface was first investigated by Nomarski differential interference contrast optical microscope, and then with an atomic force microscope (AFM) operated in the tapping mode to preserve high resolution.

The estimated fluence on the surface of the PMMA was $97 \mu \mathrm{J} / \mathrm{cm}^{2}$ at $21.6 \mathrm{~nm}, 14.7 \mathrm{~mJ} / \mathrm{cm}^{2}$ at $820 \mathrm{~nm}$, and $6.3 \mathrm{~mJ} / \mathrm{cm}^{2}$ at $410 \mathrm{~nm}$ per shot, respectively. As all these values lie far below the ablation threshold for PMMA by infrared $\left(2.6 \mathrm{~J} / \mathrm{cm}^{2}\right.$ for single-shot and $0.6 \mathrm{~J} / \mathrm{cm}^{2}$ for 100 shots - Baudach et al., 2000) as well as by XUV $\left(2 \mathrm{~mJ} / \mathrm{cm}^{2}\right.$ - Chalupsky et al., 2007) radiation, no damage of target surface was expected. 

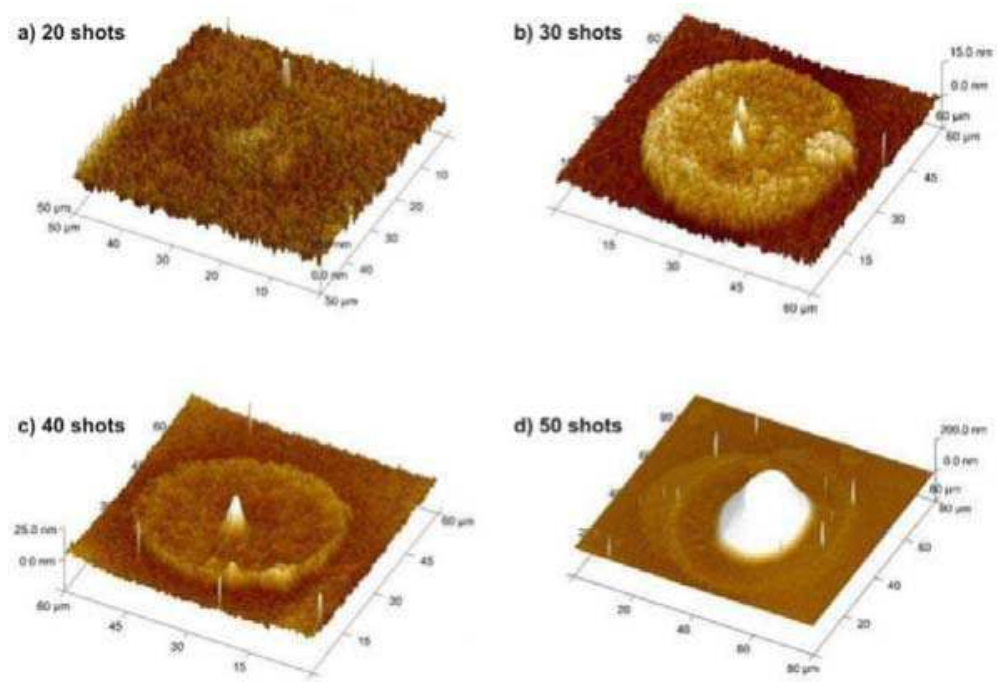

Fig. 10. AFM images of the PMMA surface simultaneously irradiated with XUV and VisNIR ultrashort pulses (Mocek et al., 2009).

The PMMA target was irradiated under the following conditions: a) Vis-NIR beams only (no gas jet in operation), b) XUV beam only $(0.4 \mu \mathrm{m}$ Al filter placed in front of the OAP), and c) mixed XUV/Vis-NIR field $(21.6 \mathrm{~nm}+820 \mathrm{~nm}+\mathrm{SH})$. In cases a) and b) we have not observed any signs of surface damage after irradiation by $\sim 3000$ shots, although the accumulated dose was significantly higher than in case c). The target surface remained significantly unaffected, preserving its original quality and roughness. In striking contrast, the application of the mixed XUV/Vis-NIR field resulted in clearly visible, irreversible surface modification after irradiation with only a few shots. The damage is characteristic due to material expansion, quite different from ablation craters observed in experiments with pure XUV pulses (Chalupsky et al., 2007; Juha et al., 2005; Vaschenko et al., 2006; Krzywinski et al., 2007; Mocek et al., 2006). A very interesting feature in Fig. 10 is the formation of nano-scale spike(s) in the center of a uniform flat pedestal. The size of pedestal increases with the number of shots applied to the target ( $\sim 20 \mu \mathrm{m}$ for 10 shots, $\sim 60 \mu \mathrm{m}$ for 50 shots) while the spikes have a radius of the order of few microns down to a few tens of nanometers in length ( $\sim 15 \mathrm{~nm}$ for 30 shots, $\sim 25 \mathrm{~nm}$ for 40 shots). The target was also analyzed by Fouriertransform infrared spectroscopy, which revealed that the expanded material is exclusively PMMA, not the silicon from substrate.

In another experiment (the same experimental setup) $890 \mathrm{~nm}$ thick amorphous carbon (a-C) sample was simultaneously irradiated by beams: $15 \mathrm{~mJ} / \mathrm{cm}^{2}$ at $820 \mathrm{~nm}, 6 \mathrm{~mJ} / \mathrm{cm}^{2}$ at $410 \mathrm{~nm}$, and $0.1 \mathrm{~mJ} / \mathrm{cm}^{2}$ at $21.6 \mathrm{~nm}$. The sample irradiated solely with NIR-VIS light exhibited only negligible surface changes, whilst in the case of combined fields the area of the modified surface dramatically increases. The damage occurred in a single pulse exposure. RMS microroughness of NIR-VIS and XUV/NIR-VIS illuminated samples was estimated to be $4.7 \mathrm{~nm}$ and $176.5 \mathrm{~nm}$, respectively (evaluated only in the interaction region), and $5.7 \mathrm{~nm}$ for the unexposed sample (Horcas et al., 2007). The efficiency of the dual action is much higher than in the previous experiment due to larger fluencies of the beams. The difference in the 
surface changes visible in AFM scans suggests that the structures shapes are fluencedependent.

Even stronger enhancement of surface processing by the dual action was observed on a-C sample. Laser induced periodic surface structures (LIPSS, or "ripples") with spatial period of $\sim 550 \mathrm{~nm}$ were created during 10-shot exposure in both cases; however, a clear difference in modulation depth was observed: the peak-to-valley depth was $\sim 130 \mathrm{~nm}$ for NIR-VIS irradiated sample, while $\sim 200 \mathrm{~nm}$ for combined XUV/NIR-VIS. Moreover, despite its random distribution, the frequency of occurrence of LIPSS is much higher in the case of the mixed fields. RMS microroughness was $13.5 \mathrm{~nm}$ for NIR-VIS illumination, $46.2 \mathrm{~nm}$ for XUV/NIR-VIS, and $8.3 \mathrm{~nm}$ for an unexposed sample, respectively.

Detailed discussion on experimental results and physical processes involved in the dual action can be found in Jakubczak et al., b) and Jakubczak et al., c).

\subsection{Multilayer optics metrology}

The high-order harmonic source was also used for reflectivity measurements of multilayer mirror (ML) versus the incidence angle. The experimental setup is presented in Fig. 11. The driving laser field featured the parameters: $\lambda_{c}=820 \mathrm{~nm}, \Delta \mathrm{t}=30 \mathrm{fs}, \mathrm{E}=4.5 \mathrm{~mJ}$. For efficient HHG generation the beam was frequency doubled with BBO crystal with conversion efficiency of $\sim 27 \%$. Subsequently, the two beams were focused by a spherical mirror ( $\mathrm{f}=125 \mathrm{~mm}$ ) into a gas cloud created by a helium gas jet at 3 bar backing pressure. The gas jet was $9 \mathrm{~mm}$ long. To filter out IR laser light $200 \mathrm{~nm}$ Al filter was used. The high-order harmonic source was optimized for the strongest harmonic line at $21.6 \mathrm{~nm}$. The mirror comprised molybdenumsilicon multilayer structure deposited on BK7 substrate. It was designed for operation at $45^{\circ}$ and $21.6 \mathrm{~nm}$ wavelength. The mirror was placed on the rotational stage together with the XUV sensitive absolutely calibrated photodiode allowing change of the incidence angle $\beta$ under vacuum. The photodiode is additionally covered with $150 \mathrm{~nm}$ Al filter. The signal was acquired with an oscilloscope.

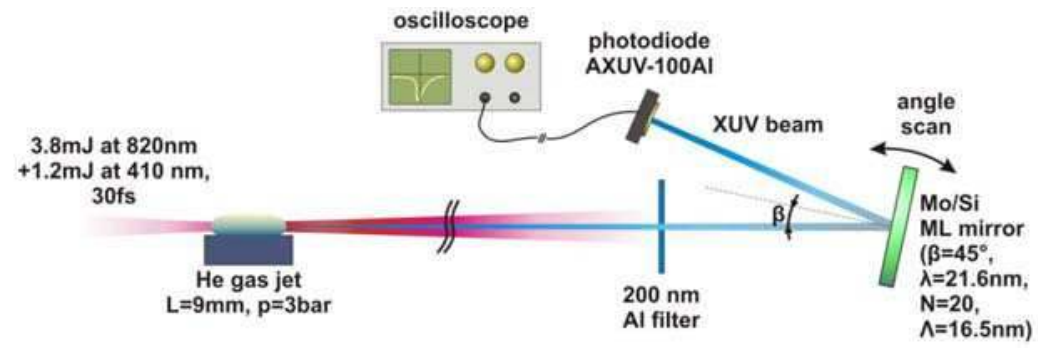

Fig. 11. Experimental setup of measurement of reflectivity of multilayer mirror versus incidence angle (Jakubczak c)).

Experimental results are presented in Graph 1. The measured and simulated reflectivity for the mirror are presented. The measured reflectivity was $31.5 \%$ (fitted value) and simulated $\sim 29 \%$. The insignificant difference could be due to the imperfections of manufacturing process as well as because of the possible additional errors (e.g. source instability or estimation error when taking into account additional input from neighboring harmonics). 


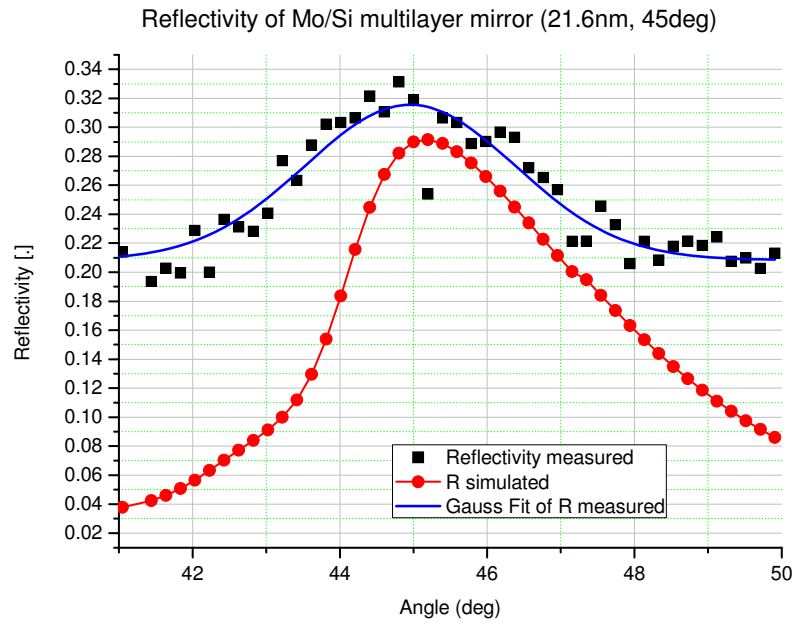

Graph 1. Reflectivity measurement of Mo/Si flat multilayer mirror designed for $21.6 \mathrm{~nm}$ radiation incident at $45^{\circ}$ angle.

\section{Conclusion}

High-order harmonics from their first observation have been extensively investigated resulting in a good understanding of physical processes involved in their generation. The main physical processes can now be well understood on the basis of the three step model proposed by P. B. Corkum (Corkum, 1993). Due to the great advent in the ultrafast lasers technology, impressive progress has been recently observed in the efficiency of HHG, cutoff extension to shorter-wavelength region (even to hard X-rays) and generation of attosecond pulses. This also allowed increase of energy of XUV pulses obtained from HHG by means of two-color HHG as well as amplification of HHG in XRL plasma amplifiers. Having at hand sub- $\mu$ J pulses of coherent pico- and femtosecond XUV pulses it became feasible to find practical applications of the source. Some of them cover investigation of ultrafast processes in atoms, others; however, are of very practical use in, e.g., structuring of surface of the materials or multilayer optics metrology. Future perspectives for HHG sources are very promising. Additionally, there is lots of activity observed in the generation of harmonics from vacuum-solid interface. Both techniques require; however, further improvements in femtosecond laser technology - especially in terms of pulse intensity and pulse contrast. For instance, efficient HHG from plasma at the interface of the vacuum and solid target intensities higher than $10^{17} \mathrm{~W} / \mathrm{cm}^{2}$ are required with the pulse contrast greater than $10^{6}$. Moreover, generation of single attosecond pulses requires carrier-envelope stabilization of the driving laser field. Finally, in order to extend cut-off in HHG from gases there are built femtosecond laser systems in the mid-infrared spectral region what found its motivation from the fact that cut-off spectral position is proportional to $I \cdot \lambda^{2}$ (Eq. 3).

The HHG source is assessed to be a very promising for needs of the future ultra compact sources of coherent radiation in XUV, soft X-ray and even X-ray spectral regions. It should 
be stressed that at the moment there is no other source of short-wavelength coherent radiation and of ultrashort pulse duration at the same time. The source would be also available at laboratory scale providing an unprecedented tool for investigation of the ultrashort physical processes. It should be stated; however, that high-order harmonics cannot substitute every kind of XUV radiation source. As an example, this type of the source is not of practical use in plasma backlighting.

\section{Acknowledgments}

This work was partially supported by the Czech Science Foundation (grants 202/07/J008, 202/08/1734), by the Czech Ministry of Education, Youth and Sports (project 7E09113), and by the Academy of Sciences of the Czech Republic (projects KAN300100702, M100100911).

\section{References}

Ammosov, M. V., Delone, N. B., Krainov, V. P., (1986). Sov. Phys. - JETP, Vol. 64, 1191.

Balcou, P., Haroutunian, R., Sebban, S., Grillon, G., Rousse, A., Mullot, G., Chambaret, J.-P., Rey, G., Antonetti, A., Hulin, D., Roos, L., Descamps, D., Gaarde, M. B., L'Huillier, A., Constant, E., Mevel, E., von der Linde, D., Orisch, A., Tarasevitch, A., Teubner, U., Klöpfel, D., and Theobald, W., (2002). High-order-harmonic generation: towards laser-induced phase-matching control and relativistic effects, Appl. Phys. B, Vol. 74, pp. 509-515.

Baltuska, A., Udem, Th., Uiberacker, M., Hentschel, M., Goulielmakis, E., Gohle, Ch., Holzwarth, R., Yakovlev, V. S., Scrinzi, A., Hänsch, T. W., \& Krausz, F. (2003). Attosecond control of electronic processes by intense light fields", Nature, Vol. 421, pp. 611-615.

Baudach, S., Bonse, J., Krüger, J., Kautek, W., (2000). Ultashort pulse laser ablation of polycarbonate and polymethylmethacrylate, Appl. Surf. Sci., Vol. 154-155, pp. 555560.

Becker, U., and Shirley, D. A., (1996). VUV and Soft X-Ray Photoionization, Plenum, New York.

Bezzerides, B., Jones, R. D., and Forslund, D. W., (1982). Plasma Mechanism for Ultraviolet Harmonic Radiation Due to Intense $\mathrm{CO}_{2}$ Light, Phys. Rev. Lett., Vol. 49, No. 3, pp. 202 - 205 (1982).

Boyd, R. W., (2003). Nonlinear Optics, Second Edition, Academic Press.

Brabec, T. and Krausz, F., (2000). Intense few-cycle laser fields: Frontiers of nonlinear optics, Rev. Mod. Phys., Vol. 72, No. 2, pp. 545-591.

Carman, R. L., Forslund, D. W., and Kindel, J. M., (1981). Visible Harmonic Emission as a Way of Measuring Profile Steepening, Phys. Rev. Lett., Vol. 46, No. 1, pp. 29 - 32.

Chalupsky, J., Juha, L., Kuba, J., Cihelka, J., Hakova, V., Koptyaev, S., Krasa, J., Velyhan, A., Bergh, M., Caleman, C., Hajdu, J., Bionta, R. M., Chapman, H., Hau-Riege, S. P., London, R. A., Jurek, M., Krzywinski, J., Nietubyc, R., Pelka, J. B., Sobierajski, R., Meyer-ter-Vehn, J., Krenz-Tronnier, A., Sokolowski-Tinten, K., Stojanovic, N., Tiedtke, K., Toleikis, S., Tschentscher, T., Wabnitz, H., and Zastrau, U., (2007). Characteristics of focused soft X-ray free-electron laser beam determined by ablation of organic molecular solids, Opt. Express, Vol. 15, 6036. 
Christov, P., Murnane, M. M., and Kapteyn, H. C., (1997). High-Harmonic Generation of Attosecond Pulses in the "Single-Cycle" Regime, Phys. Rev. Lett., Vol. 78, No. 7, pp. $1251-1254$.

Corkum, P. B., Burnett, N. H., and Brunel, F., (1989). Above-threshold ionization in the longwavelength limit, Phys. Rev. Lett., Vol. 62, No. 11, pp. 1259 - 1262 (1989).

Corkum, P. B., (1993). Plasma perspective on strong field multiphoton ionization, Phys. Rev. Lett., Vol. 71, No. 13, 1994-1997.

Drescher, M., Hentschel, M., Kienberger, R., Uiberacker, M., Yakovlev, V., Scrinzi, A., Westerwalbesloh, Th., Kleineberg, U., Heinzmann, U., \& Krausz, F., (2002). Timeresolved atomic inner-shell spectroscopy, Nature, Vol. 419, pp. 803-807.

Dromey, B., Zepf, M., Gopal, A., Lancaster, K., Wei, M. S., Krushelnick, K., Tatarakis, M., Vakakis, N., Moustaizis, S., Kodama, R., Tampo, M., Stoeckl, C., Clarke, R., Habara, H., Neely, D., Karsch, S., and Norreys, P., (2006). High harmonic generation in the relativistic limit, Nature Physics, Vol. 2, pp. 456 - 459.

Hay, N., Velotta, R., Lein, M., de Nalda, R., Heesel, E., Castillejo, M., and Marangos, J. P., (2002). High-order harmonic generation in laser-aligned molecules, Phys. Rev. A, Vol. 65, 053805.

Hentschel, M., Kienberger, R., Spielmann, Ch., Reider, G. A., Milosevic, N., Brabec, T., Corkum, P., Heinzmann, U., Drescher, M., Krausz, F., (2001). Attosecond metrology, Nature, Vol. 414, pp. 509-513.

Horcas, I., Fernandez, R., Gomez-Rodriguez, J. M., Colchero, J., Gomez-Herrero, J., and Baro, A. M., (2007). WSxM Software, Rev. Sci. Instrum., Vol. 78 , 013705.

Itatani, J., Quéré, F., Yudin, G. L., Ivanov, M. Yu., Krausz, F., and Corkum, P. B., (2002). Attosecond Streak Camera, Phys. Rev. Lett., Vol. 88, No. 17, 173903-1.

Itatani, J., Levesque, J., Zeidler, D., Niikura, H., Pépin, H., Kieffer, J. C., Corkum, P. B., and Villeneuve, D. M., (2004). Tomographic imaging of molecular orbitals, Nature, Vol. 432, pp. 867-871.

Jaeglé, P., (2006). Coherent Sources of XUV Radiation. Soft X-Ray Lasers and High-Order Harmonic Generation, Springer.

Jakubczak, K., a)- Doctoral Thesis.

Jakubczak, K., Mocek, T., Chalupsky, J., Lee, G. H., Kim, T. K., Park, S. B., Nam, Ch. H., Hajkova, V., Juha, L., and Rus, B., b). Enhanced surface structuring by XUV/NIR ultrafast dual action - in preparation.

Jakubczak, K., Mocek, T., Rus, B., Polan, J., Hrebicek, J., Sawicka, M., Sobota, J., Fort, T., Pina, L., c). Beam properties of fully optimized, table-top, coherent source at $30 \mathrm{~nm}$ - Optoelectronics Review - accepted.

Juha, L., Bittner, M., Chvostova, D., Krasa, J., Kozlova, M., Pfeifer, M., Polan, J., Präg, A. R., Rus, B., Stupka, M., Feldhaus, J., Letal, V., Otcenasek, Z., Krzywinski, J., Nietubyc, R., Pelka, J. B., Andrejczuk, A., Sobierajski, R., Ryc, L., Boody, F. P., Fiedorowicz, H., Bartnik, A., Mikolajczyk, J., Rakowski, R., Kubat, P., Pina, L., Horvath, M., Grisham, M. E., Vaschenko, G. O., Menoni, C. S., and Rocca, J. J., (2005). Shortwavelength ablation of molecular solids: pulse duration and wavelength effects, $J$. Microlith. Microfab. Microsyst., Vol. 4, 033007.

Kanai, T., Minemoto, S., and Sakai, H., (2007). Ellipticity Dependence of High-Order Harmonic Generation from Aligned Molecules, Phys. Rev. Lett. Vol. 98, 053002.

Keldysh, L. V., (1965). Sov. Phys. - JETP, Vol. 20, 1307. 
Kienberger, R., Hentschel, M., Uiberacker, M., Spielmann, Ch., Kitzler, M., Scrinzi, A., Wieland, M., Westerwalbesloh, Th., Kleineberg, U., Heinzmann, U., Drescher, M., Krausz, F., (2002). Steering Attosecond Electron Wave Packets with Light, Science, Vol. 297, pp. 1144-1148 (2002).

Kienberger, R., Goulielmakis, E., Uiberacker, M., Baltuska, A., Yakovlev, V., Bammer, F., Scrinzi, A., Westerwalbesloh, Th., Kleineberg, U., Heinzmann, U., Drescher M. \& Krausz F., (2004). Atomic transient recorder, Nature, Vol. 427, pp. 817-821.

Kienberger, R., Uiberacker, M., Kling, M. F., Krausz, F., (2007). Attosecond physics comes of age: from tracing to steering electrons at sub-atomic scales, J. Modern Optics, Vol. 54, No. 13-15, pp. 1985-1998.

Kim, I J., Kim, C. M., Kim, H. T., Lee, G. H., Lee, Y. S., Park, J. Y., Cho, D. J., and Nam, Ch. H., (2005). Highly Efficient High-Harmonic Generation in an Orthogonally Polarized Two-Color Laser Field, Phys. Rev. Lett., Vol. 94, 243901.

Kim, I J., Lee, G. H., Park, S. B., Lee, Y.S., Kim, T. K., Nam, Ch. H., Mocek, T., and Jakubczak, K., (2008). Generation of submicrojoule high harmonics using a long gas jet in a two-color laser field, Appl. Phys. Lett., Vol. 92, 021125.

Krzywinski, J., Sobierajski, R., Jurek, M., Nietubyc, R., Pelka, J. B., Juha, L., Bittner, M., Letal, V., Vorlicek, V., Andrejczuk, A., Feldhaus, J., Keitel, B., Saldin, E., Schneidmiller, E., Treusch, R., and Yurkov, M., (2007). Conductors, semiconductors, and insulators irradiated with short-wavelength free-electron laser, J. Appl. Phys., Vol. 101, 043107.

L'Huillier, A., and Balcou, Ph., (1993). High-order harmonic generation in rare gases with a 1-ps 1053-nm laser, Phys. Rev. Lett., Vol. 70, No. 6, pp. 774 - 777.

Lorin, E., Chelkowski, S., and Bandrauk, A. D., (2008). Attosecond pulse generation from aligned molecues - dynamics and propagation in $\mathrm{H}^{2+}$, New Journal of Physics, Vol. 10, 025033.

Macklin, J. J., Kmetec, J. D., Gordon, C. L., (1993). High-order harmonic generation using intense femtosecond pulses, Phys. Rev. Lett., Vol. 70, No. 6, pp. 766-769.

Mairesse, Y., and Quéré, F., (2005). Frequency-resolved optical gating for complete reconstruction of attosecond bursts, Phys Rev. A, Vol. 71, pp. 1-4, 011401.

Mocek, T., Rus, B., Kozlova, M., Stupka, M., Präg, A. R., Polan, J., Bittner, M., Sobierajski, R., and Juha, L., (2006). Focusing a multimillijoule soft x-ray laser at $21 \mathrm{~nm}$, Appl. Phys. Lett., Vol. 89, 051501.

Mocek, T., Polan, J., Homer, P., Jakubczak, K., Rus, B., Kim, I J., Kim, C. M., Lee, G. H., Nam, Ch. H., Hajkova, V., Chalupsky, J., and Juha, L., (2009). Surface modification of organic polymer by dual action of extreme ultraviolet/visible-near infrared ultrashort laser pulses, J. Appl. Phys., Vol. 105, 026105.

Niikura, H., Légaré, F., Hasbani, R., Bandrauk, A. D., Ivanov, M. Yu., Villeneuve, D. M., and Corkum, P. B., (2002). Sub-laser-cycle electron pulses for probing molecular dynamics, Nature, Vol. 417, pp. 917-922.

Papadogiannis, N. A., Witzel, B., Kalpouzos, C., and Charalambidis, D., (1999). Observation of Attosecond Light Localization in Higher Order Harmonic Generation, Phys. Rev. Lett., Vol. 83, No. 21, pp. 4289 - 4292.

Paul, P. M., Toma, E. S., Breger, P., Mullot, G., Augé, F., Balcou, Ph., Muller, H. G., Agostini P., (2001). Observation of a Train of Attosecond Pulses from High Harmonic Generation, Science, Vol. 292, pp. 1689-1692.

Pfeifer, T., Spielmann, C., and Gerber, G., (2006). Femtosecond x-ray science, Rep. Prog. Phys., Vol. 69, pp. 443-505. 
Quéré, F., Thaury, C., Monot, P., Dobosz, S., and Martin, Ph., (2006). Coherent Wake Emission of High-Order Harmonics from Overdense Plasmas, Phys. Rev. Lett., Vol. 96, 125004-1.

Quéré, F., Thaury, C., Geindre, J-P., and Martin, Ph., (2008). Comment on “Transition to the Relativistic Regime in High Order Harmonic Generation", Phys. Rev. Lett. Vol. 100, 089401.

Remetter, T., Johnsson, P., Mauritsson, J., Varjú, K., Ni, Y., Lépine, F., Gustafsson, E., Kling, M., Khan, J., López-Martens, R., Schafer, K. J., Vrakking, M. J. J. and L'Huillier, A., (2006). Attosecond electron wave packet interferometry, Nature Physics, Vol. 2, pp. $323-326$.

Roentgen, W. C., (1896). On a new kind of rays, Nature, Vol. 53, pp. 274-276.

Rundquist, A., Durfee, Ch. G., Chang, Z., Herne, C., Backus, S., Murnane, M. M., Kapteyn, H. C. (1998). Phase-Matched Generation of Coherent Soft X-rays, Science, Vol. 280, pp. 1412 - 1415.

Sakai, H., Miyazaki, K., (1995). High-order harmonic generation in nitrogen molecules with subpicosecond visible dye-laser pulses, Appl. Phys. B, Vol. 61, pp. 493-498 (1995).

Sansone, G., Benetti, E., Vozzi, C., Stagira, S., and Nisoli, M., (2008). Attosecond metrology in the few-optical-cycle regime, New Journal of Physics, Vol. 10, 025006.

Schenkel, B., Biegert, J., Keller, U., Vozzi, C., Nisoli, M., Sansone, G., Stagira, S., De Silvestri, S., and Svelto, O., (2003). Generation of 3.8-fs pulses from adaptive compression of a cascaded hollow fiber supercontinuum, Opt. Lett., Vol. 28, No. 20, pp. 1987-1989 (2003).

Schultze, M., Goulielmakis, E., Uiberacker, M., Hofstetter, M., Kim, J., Kim, D., (2007). Powerful 170-attosecond XUV pulses generated with few-cycle laser pulses, New Journal of Physics, Vol. 9, Vol. 243, pp. 1-11.

Sola, J., Mével, E., Elouga, L., Constant, E., Strelkov, V., Poletto, L., Villoresi, P., Benedetti, E., Caumes, J.-P., Stagira, S., Vozzi, C., Sansone, G., and Nisoli M., (2006). Controlling attosecond electron dynamics by phase-stabilized polarization gating, Nature Physics, Vol. 2, pp. 319 - 322.

Tarasevitch, A., Lobov, K., Wünsche, C., and von der Linde, D., (2007). Transition to the Relativistic Regime in High Order Harmonic Generation, Phys. Rev. Lett., Vol. 98, 103902.

Thaury, C., Quéré, F., Geindre, J.-P., Levy, A., Ceccotti, T., Monot, P., Bougeard, M., Réau, F., d'Oliveira, P., Audebert, P., Marjoribanks, R., \& Martin, Ph., (2007). Plasma mirrors for ultrahigh-intensity optics, Nature Physics, Vol. 3, pp. 424 - 429.

Vaschenko, G., Etxarri, A. G., Menoni, C. S., Rocca, J. J., Hemberg, O., Bloom, S., Chao, W., Anderson, E. H., Attwood, D. T., Lu, Y., and Parkinson, B., (2006). Nanometer-scale ablation with a table-top soft x-ray laser, Opt. Lett., Vol. 31, 3615.

Velotta, R., Hay, N., Mason, M. B., Castillejo, M., and Marangos, J. P., (2001). High-Order Harmonic Generation in Aligned Molecules, Phys. Rev. Lett., Vol. 87, 183901.

Véniard, V., Taïeb, R., and Maquet, A., (1996). Phase dependence of (N+1)-color (N > 1) iruv photoionization of atoms with higher harmonics, Phys. Rev. A, Vol. 54, No. 1, pp. 721-728 (1996). 


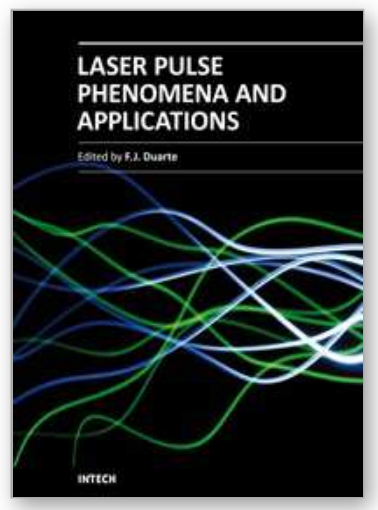

\author{
Laser Pulse Phenomena and Applications \\ Edited by Dr. F. J. Duarte
}

ISBN 978-953-307-405-4

Hard cover, 474 pages

Publisher InTech

Published online 30, November, 2010

Published in print edition November, 2010

Pulsed lasers are available in the gas, liquid, and the solid state. These lasers are also enormously versatile in their output characteristics yielding emission from very large energy pulses to very high peak-power pulses.

Pulsed lasers are equally versatile in their spectral characteristics. This volume includes an impressive array of current research on pulsed laser phenomena and applications. Laser Pulse Phenomena and Applications covers a wide range of topics from laser powered orbital launchers, and laser rocket engines, to laser-matter interactions, detector and sensor laser technology, laser ablation, and biological applications.

\title{
How to reference
}

In order to correctly reference this scholarly work, feel free to copy and paste the following:

Krzysztof Jakubczak (2010). High-order Harmonic Generation, Laser Pulse Phenomena and Applications, Dr. F. J. Duarte (Ed.), ISBN: 978-953-307-405-4, InTech, Available from: http://www.intechopen.com/books/laserpulse-phenomena-and-applications/high-order-harmonic-generation

\section{INTECH}

open science | open minds

\section{InTech Europe}

University Campus STeP Ri

Slavka Krautzeka 83/A

51000 Rijeka, Croatia

Phone: +385 (51) 770447

Fax: +385 (51) 686166

www.intechopen.com

\section{InTech China}

Unit 405, Office Block, Hotel Equatorial Shanghai

No.65, Yan An Road (West), Shanghai, 200040, China

中国上海市延安西路65号上海国际贵都大饭店办公楼 405 单元

Phone: +86-21-62489820

Fax: $+86-21-62489821$ 
(C) 2010 The Author(s). Licensee IntechOpen. This chapter is distributed under the terms of the Creative Commons Attribution-NonCommercialShareAlike-3.0 License, which permits use, distribution and reproduction for non-commercial purposes, provided the original is properly cited and derivative works building on this content are distributed under the same license. 